\title{
Drug Receptor Interactions, Metabolism, and Preclinical Drugs for SARS COV 2
}

\author{
Angayarkanni Thirumalai, Chalos Angel Varghese, Harsha N, Deepika J. \\ Department of Biochemistry, Biotechnology, and Bioinformatics, Avinashilingam Institute for Home Science and Higher Education for \\ Women, Coimbatore- 641 043, Tamil Nadu, India. \\ *Corresponding author's E-mail: angait73@gmail.com
}

Received: 05-11-2020; Revised: 17-12-2020; Accepted: 28-12-2020; Published on: 15-01-2021.

\begin{abstract}
Drugs by definition are categorized as those agents that can bring about a change in any living species and habitually used for therapeutic purposes. Mostly majority of drugs show an extraordinarily high correlation of structure and specificity to produce pharmacological effects. A large classification of them put forth their physiologic effects by binding with a naturally selective receptor and thus making the drug-receptor interaction an extensively studied subject, bearing in mind the complex intracellular biochemistry, pharmacology, and energetics involved in the entire event. Experimental studies specify that drugs interact with receptor sites localized in macromolecules that have got protein-like properties and also specific three-dimensional shapes. Drug metabolism is an important process for the removal of unwanted substances from the body. An abnormal drug metabolism profile could lead to lifethreatening complications. Both phase I (mainly CYP450s) and phase II (mainly UGTs) enzymes play a significant role in drug metabolism. Although metabolites, in general, are expected to be not active and not toxic, certain metabolites can cause hepatotoxicity. Various diseases may potentially change the metabolic profile of a drug by altering the expression and function of key enzymes. Additionally, coadministration of multiple drugs may also lead to drug-drug interaction and adverse reactions due to competitive binding to the same metabolizing enzyme. Currently, SARS Cov 2 is an illness ongoing worldwide. This virus is responsible for severe respiratory illness and nearly 2,500,000 people worldwide have been diagnosed with SARS-CoV-2. No vaccine or antiviral drugs are available currently to treat SARS-CoV-2. Different pre-clinical studies suggested that hydroxychloroquine and chloroquine, arbidol, remdesivir, lopinavir/ritonavir, ivermectin, tocilizumab, ribavirin, and anti-inflammatory drugs can show a promising outcome for SARS-Cov-2. This review article focuses on the drug-receptor interaction, drug metabolism, and preclinical drugs for SARS Cov 2.
\end{abstract}

Keywords: Drug interaction, GPCRs, Drug metabolism, Cytochrome P450, Drug transporters, Liver metabolism, SARS-CoV-2, Arabinol, Remdesivir, Chloroquine, and hydroxychloroquine.

QUICK RESPONSE CODE $\rightarrow$

DOI:

10.47583/ijpsrr.2021.v66i01.013

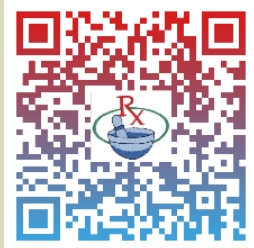

DOI link: $\underline{\text { http://dx.doi.org/10.47583/ijpsrr.2021.v66i01.013 }}$

\section{INTRODUCTION}

T

he receptor theory of drug action theorizes that a drug only works when bound to its target receptor ${ }^{1}$. Until the theory was first postulated the direct measurement of the extent to which a drug is bound to its receptor at equilibrium the binding affinity was, not possible. Consequently, drug discovery programs historically sought to enhance drug efficacy, not affinity, generally in the background of whole cells, tissues, or animals. With the arrival of identifiable, and ultimately purifiable, molecular receptors, the direct measurement of binding affinity, optimization of binding affinity guide most early-stage discovery efforts ${ }^{2-4}$. The interaction between drugs and receptors was based on Michaelis Menten kinetics. They are utilized to create the quantitative tools presently used in receptor pharmacology, which help to quantify drug effect in biological systems. Those tools are essential as drugs practically always are tested in surrogate systems until they are known to be adequately active and safe for therapeutic use or application. The main objective of receptor pharmacology is to describe the molecular properties of affinity and efficacy of drugs and it has got a wide application, these can be used to foresee drug effect through altered biological systems.

The term "metabolism" refers to the process of transformation of chemicals from one chemical moiety to another by an enzyme. The most well-known drugmetabolizing enzymes are cytochrome P450s(CYP450s), which are mainly oxidases, reductases, and hydrolases ${ }^{5}$. The primary purpose of metabolism is to clear endogenous and/or exogenous molecules from the body. Typically, the process of metabolism converts lipophilic chemicals to hydrophilic products to facilitate elimination. Drug metabolizing enzymes convert substances into their pharmacologically active form. The by-products of metabolism are known as metabolites; they can be either pharmacologically active or inactive ${ }^{6}$. CYP450 enzymes that play a major role in drug elimination are mainly present on the smooth endoplasmic reticulum (ER) and mitochondria of the hepatocytes and small intestinal epithelia and to a lesser extent in the proximal tubules of the kidneys. The contribution and importance of 
conjugating enzymes and drug transporters are increasingly appreciated. These pathways interplay during the absorption, distribution, metabolism, and excretion of drugs, and any alterations may result in changes in the pharmacokinetics and pharmacodynamics of a drug ${ }^{5,6}$.

Coronavirus is a single-stranded RNA virus. They can infect humans and animals causing respiratory, gastrointestinal, hepatic, and neurologic diseases. Coronavirus is further divided into alpha- coronavirus, beta-coronavirus, gammacoronavirus, and delta-coronavirus. Six human coronaviruses ( $\mathrm{HCOVs}$ ) have been identified, including the alpha-CoVs HCoVs-NL63 and HCoVs-229E and the betaCoVs HCoVs-OC43, HCoVs-HKU1, severe acute respiratory syndrome-CoV (SARS-CoV), and Middle East respiratory syndrome-CoV (MERS-CoV) ${ }^{7}$. In December 2019, adults in Wuhan started presenting to local hospitals with severe pneumonia of unknown cause. The samples of the patients were collected and investigated. On January 7th, 2020, they identified the virus as beta-coronavirus which they had never seen before and had $>95 \%$ homology with the bat coronavirus 8,9 . This new coronavirus was highly contagious. The WHO, on 30 January 2020, declared COVID-19 as a "public health emergency of international concern". The pandemic is escalating rapidly ${ }^{7}$. Currently, no specific antiviral agent is recommended to treat patients with COVID-19 and no vaccine is available. Different preclinical in vitro and in vivo studies on other CoV-induced diseases suggested that promising clinical outcomes for SARS-Cov-2 patients should be obtained by using hydroxychloroquine and chloroquine, arbidol, remdesivir, lopinavir/ritonavir, ivermectin, tocilizumab, ribavirin, and anti-inflammatory drugs. More clinical trials should be performed on patients affected by COVID-19 with these drugs to test their efficacy and safety as recently proposed for tocilizumab ${ }^{10}$. This review focuses on the drug-receptor interaction, drug metabolism, and preclinical drugs for SARS-CoV-2.

\section{Role of receptor in drug-receptor interaction}

The receptors are macromolecules that are involved in chemical signaling within and also between the cells. They may be situated on the cell surface membrane or within the cytoplasm. Molecules such as drugs, hormones, neurotransmitters that bind to a receptor are called ligands. The binding can be specific and reversible. A ligand may activate or can inactivate a receptor. Activated receptors regulate cellular biochemical processes directly or indirectly and they include ion conductance, protein phosphorylation, DNA transcription, enzymatic activity. Activation of the ligand may increase or decrease a specific cell function. Every single ligand may interact with multiple receptor subtypes, few if any drugs are specific for one receptor or subtype, but most of them have relative selectivity. Selectivity is very vital, to which a drug acts on a given site relative to other sites. Selectivity is related mostly to the physicochemical binding of the drug to cellular receptors. A drug's ability to affect a given receptor is related to the drug's affinity that is the possibility of the drug occupying a receptor at any given instant and also intrinsic efficacy that is the degree to which a ligand activates receptors and which leads to a cellular response. Through the chemical structure of drugs, its affinity and activity are determined. The pharmacologic effect can also be confirmed by the duration of time that the drug-receptor complex sticks at, and they can also be said as residence time. The period of the drug-receptor complex is affected by vibrant processes which control the rate of drug association and dissociation from the target. A longer residence time elucidates a prolonged pharmacological effect. Longer residence time can be a possible drawback when it prolongs a drug's toxicity. For particular receptors, transient drug occupancy produces the desired pharmacologic effect, although prolonged occupancy causes toxicity ${ }^{11}$.

\section{Theory of receptor}

Living organisms interconnect with various chemical compounds through receptors, which is described by the receptor theory. The affinity or the attraction of the biologically active compounds toward receptors and their ability to trigger or activate a biological or toxic signal differ substantially. The theory or the concept of the receptor was independently proposed by Ehrlich and Langley at the beginning of the 20th century ${ }^{12}$.In this work or concept, we describe a new insight into the understanding of the mode of action of receptor partial agonists and the receptor theory using a Full Logistic Model (FLM) of mixture toxicology.

The main aim of this was to explain the selective effects of drugs and it also suggested the action of a drug-involved during the formation of specific complexes with help of molecular agents in the target cells, thus stimulating a cell response. We describe the hypothesis that the effect of a partial agonist can be mathematically described via the separation of agonistic and antagonistic behavior of the partial agonist where the antagonistic effect is described as an action of a compound generating zero effect. In this way, a competitive antagonist can be considered as an agonist with zero effect. This idea is also placed in a context with classical concepts, e.g., Gaddum's equation. Using the assumption that competitive antagonists are agonists with no effect, equations describing the microscopic and macroscopic equilibrium constants have been derived. Accordingly, we show that the constants could be calculated from the measured partial agonistic doseresponse curve. As a consequence, we provide a simple mathematical tool for comparison of dose-response curves of drugs according to their affinities and efficacies. In the decades that shadowed, this hypothesis was proved, receptor molecules were biochemically identified or recognized, and their structures were discovered, as a result of which enabled us to fully understand the vital role that they played in physiology. Nearly more than $4 \%$ of the human genome encodes or translates cell receptors ${ }^{13}$. These are prearranged into different families and they 
include matrix receptors (example integrins), ligand-gated (LGIC, which has 76 members in the human genome) and voltage-gated (VGIC, which has 143 members) ion channels, intracellular receptors which include nuclear hormone receptors (NHRs, which has 48 members), enzyme-linked receptors, like receptor tyrosine kinases (RTKs, which has 58 members), and G protein-coupled receptors (GPCRs) ${ }^{14}$. GPCRs contribute to the largest family in mammals, they help in almost all physiological processes, and currently, they are very common targets for drugs ${ }^{13,15}$. The GPCR family is made up of about 800 receptors in humans and they are classified into five major groups, namely classes A (the largest group), B, C, frizzled, and adhesion, which is mainly based on their structural and functional similarities ${ }^{16,17}$. GPCRs have a highly conserved overall structure ${ }^{18,19}$, revealing seven $\alpha$-helixes that span the plasma membrane-Transmembrane domains, TM, and they are connected by extra- and an intracellular loops like ECL and ICL. The stability of the TM region is provided by interhelical bonds and also hydrophobic interactions between highly conserved residues. The extracellular domain surrounding the $\mathrm{N}$-terminus of the protein shows high structural variability amongst the different classes of GPCRs since they being very large in class C- GPCRs ${ }^{20}$. In quite a lot of GPCRs for instance class C GPCRs, it is the domain that hosts the ligand-binding site, while in others, like most of class A GPCRs the ligand-binding pocket is positioned in the extracellular half of the TM bundle ${ }^{21}$. When ligand binding takes place, it brings a conformational change of the TM core, permitting the activation of downstream signaling pathways. In vitro and in vivo experiments, they have demonstrated that GPCRs can recognize and decode signals (of chemical or physical nature) as monomers. Studies of particular interest have shown that monomers of three class A GPCRs specifically rhodopsin, $\beta_{2}$-adrenergic, and $\mu$-opioid receptors trapped inside nanodiscs can signal 22,23. Moreover, intrinsic plasticity has been found to describe signaling from GPCR monomers, in that they can take up multiple active conformations because of their binding with ligands, thus initiating different patterns of signal transduction, ${ }^{24}$ such as $\mathrm{G}$ protein or arresting pathways ${ }^{25}$.

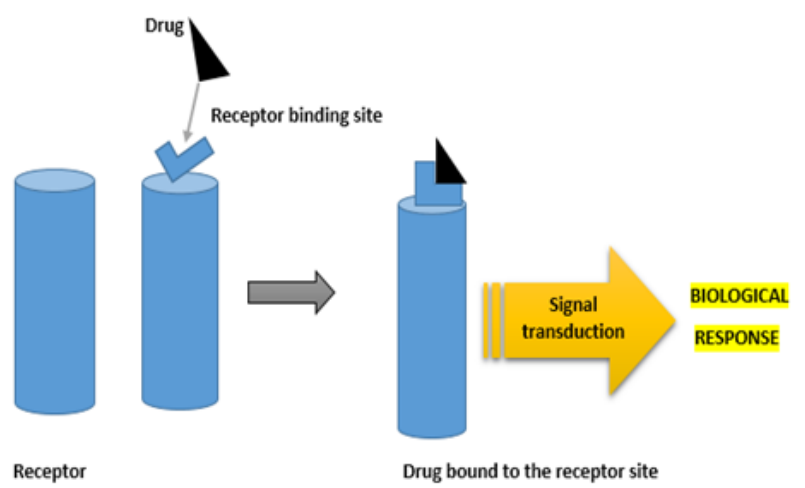

Figure 1: The interaction of drug to receptor site. When drug get bound to the receptor site, confirmational changes occurs and signal transduction takes place and their occurs a biological response ${ }^{26}$.

\section{Types of receptors}

Based on molecular structure and the nature of the transduction mechanism the receptors are classified into 4 types of receptors:

\section{Ligand-gated ion channels}

\section{G-protein-coupled receptors}

\section{Receptor kinases}

\section{Nuclear receptors ${ }^{27}$.}

\section{Physiological functions of the receptor}

Physiologic functions that area contraction, secretion are usually regulated by multiple receptor-mediated mechanisms, and several steps like receptor-coupling, multiple intracellular 2 nd messenger substances, may be interrupted between the initial molecular drug-receptor interaction and ultimate tissue or organ response. Therefore, more than a few dissimilar drug molecules can frequently be used to produce the same desired response. The binding of a ligand brings a change in receptor configuration perturbing the entire cellular equilibrium. The energy involved in the binding process triggers a cascade of biochemical reactions, which is manifested in versatile intracellular responses leading to physiological actions. The ability of binding to a receptor is influenced by external factors also by intracellular regulatory mechanisms. The efficiency of stimulusresponse mechanisms and baseline receptor density varies from tissue to tissue. Drugs, aging, genetic mutations, and disorders can increase or decrease that is upregulated or downregulate the number and binding affinity of receptors. For instance, clonidine downregulates or decreases the affinity of alpha 2 receptors; thus, rapid withdrawal of clonidine can cause a hypertensive crisis.

Chronic therapy with help of beta-blockers up-regulates beta-receptor density. Specifically, the receptor upregulation and downregulation affect adaptation to drugs that are desensitization, tachyphylaxis, tolerance, acquired resistance, post-withdrawal super sensitivity are some of the examples ${ }^{28}$.

Ligands bind to accurate molecular regions, called recognition sites, on receptor macromolecules. The binding site for a drug may be the same as or different from that of an endogenous agonist (hormone or neurotransmitter). Agonists that bind to an adjacent site or a different site on a receptor are sometimes called allosteric agonists. Nonspecific drug binding also occurs at molecular sites, which is not designated as receptors (for example plasma proteins). Drug binding to such nonspecific sites like binding to serum proteins excludes the drug from binding to the receptor and therefore inactivates the drug. The unbound drug is available to bind to receptors and thus have an effect ${ }^{28}$. 


\section{Process interaction of drug with receptor}

A neurotransmitter has got a precise shape to fit into a receptor site and root to pharmacological response such as a nerve impulse being sent. The neurotransmitter is analogous to a substrate in an enzyme interaction. After attachment to a receptor site, the drug may either initiate a response or prevent a response from happening. A drug must be a close mimic or it must be the same as that of the neurotransmitter. An agonist is a type of drug which produces a stimulation type response. The agonist is a very close mimic or they are very similar and fit with the receptor site and which able to initiate a response. An antagonist drug is a type of drug that interacts with the receptor site and blocks or depresses the normal response or function for that receptor because it only partly fits the receptor site and cannot produce an effect. However, it blocks the site, by preventing any other agonist or the normal neurotransmitter from interacting with the receptor site $^{29}$.

\section{Agonists and Antagonists}

Agonists have a key role to activate receptors to produce the desired response. Conventional agonists upsurge the proportion of activated receptors. Inverse agonists wherein stabilize the receptor in its inactive conformation and act likewise as that to competitive antagonists. Many hormones, neurotransmitters like acetylcholine, histamine, norepinephrine, and drugs like benzodiazepines, barbiturates morphine, phenylephrine, isoproterenol, act as agonists.

Antagonists avoid receptor activation. Preventing activation has numerous effects. Antagonists raise the cellular function if they block the action of a substance that normally decreases cellular function. Antagonists decrease or reduce the cellular function if they block the action of a substance that normally increases cellular function. Receptor antagonists can be classified into 2 categories reversible or irreversible. Reversible antagonists readily dissociate or disconnect from their receptor whereas irreversible antagonists form a stable, permanent, or nearly permanent chemical bond with their receptor by process of alkylation. Pseudoirreversible antagonists gradually dissociate from their receptors ${ }^{28}$.

In the case of competitive antagonism, binding of the antagonist to the receptor prevents or helps to stop the binding of the agonist to the receptor. Whereas in noncompetitive antagonism, the agonist and antagonist can be bound concurrently or simultaneously, but antagonist binding reduces or prevents the action of the agonist. In the case of reversible competitive antagonism, the agonist and antagonist form a short-lasting bond with the receptor, and from which a steady-state midst of agonist, antagonist, and receptor is grasped. Such type of antagonism can be overcome by increasing the concentration of the agonist ${ }^{28}$. For instance, the naloxone an opioid receptor antagonist that is structurally comparable to morphine, when they are given as a dose shortly before or after morphine, it completely blocks morphine's effects. On the other hand, competitive antagonism by naloxone can be overawed by giving more morphine. Structural analogs or property of agonist molecules commonly have agonist and antagonist properties. Such types of drugs are called partial or lowefficacy agonists, or agonist-antagonists. For example, pentazocine activates opioid receptors but consequently, it blocks their activation by other opioids. Therefore, pentazocine provides opioid effects but it blunts the effects of another opioid if the opioid is given during pentazocine is still bound. A drug that acts as a partial agonist in one tissue could act as a full agonist in another tissue ${ }^{28}$.

\section{Drug metabolism pathways}

\section{Phase I Pathway}

The most common phase I drug-metabolizing enzymes are represented by the CYP450 superfamily. CYP450s are a major group of enzymes that chemically modify drugs into their water-soluble products to facilitate the excretion by the kidney and/or liver. In the late 1980s, Nebert developed and reported a nomenclature system for CYP450 enzymes. Human CYP450 genes comprise more than 115 gene and pseudogene members and are among the most extensively annotated mammalian genes, starting from CYP1A1 and currently ending with CYP51P3 ${ }^{30}$. In humans, CYP450s are distributed throughout various tissues and organs, including peripheral blood cells, platelets, aorta, adrenal glands, adipose tissues, nasal tissue, vaginal tissues, seminal vesicles, brain, lung, kidneys, gut, and liver. Of all the various tissues, the liver and small intestine

contribute to the maximum extent to the overall metabolism and elimination of drugs. Among all the CYP450 enzymes are in the human liver, CYP3A4 is the most abundant, followed by CYP2E1 and CYP2C9. CYP450 enzymes also may be classified based on their major substrates, such as sterols, xenobiotics, fatty acids, eicosanoids, vitamins, and unknown substrates ${ }^{31}$. CYP450 enzymes catalyze several reactions, including oxidation, sulphoxidation, aromatic hydroxylation, aliphatic hydroxylation, N-dealkylation, O-dealkylation, and deamination. Among all, oxidation is the primary reaction, which leads to the addition of 1 or more oxygen atom(s) to the parent drug. The reduction of the parent compounds is another pathway of phase I drug metabolism ${ }^{30,31}$. This type of reaction is coupled with a secondary enzymatic system that is known to be either NADH cytochrome-b5 reductase system or NADPH cytochrome-c reductase. This route is important for metabolizing aromatic nitro, nitroso, azo, and $\mathrm{N}$-oxide compounds. CYP450s expression is regulated in different compartments of the cell, nuclei, or cytosol by many factors. Nuclear receptor-mediated regulation of gene expression occurs in the nucleus, which is the most critical regulatory pathway, resulting in differential gene transcription. Aryl hydrocarbon receptor 
is a receptor activated by several endogenous and exogenous ligands, which activates the gene translation and synthesis of various CYP450s ${ }^{31}$.

In the cytosol, cofactors, such as NADPH-Cytochrome P450 reductase; cytochrome-b5 reductase, and/or cytochromec reductase, are essential to carry out the biotransformation reactions ${ }^{30}$. Iron is important for CYP450s synthesis and is present in the center of the binding site between the enzyme and substrate. Differences in the pharmacologic responses to the same dose of a drug may result due to differences in metabolism and elimination of drugs. Apart from CYP450 enzymes, other phase I enzymes can contribute to the clearance of many drugs. Some examples of non-CYP450 enzymes that could metabolize endogenous molecules and xenobiotics include flavin-containing monooxygenases, monoamine oxidases, molybdenum hydroxylases, alcohol dehydrogenases, aldehyde dehydrogenases, aldoketoreductase, NADPH: quinone reductases, and hydrolytic enzymes ${ }^{32,33}$.

The expression and activity of CYP450 enzymes can be modulated by several factors.

Increased mRNA expression leads to increased protein synthesis and a corresponding increase in the activity of enzymes. Induction of CYP450 enzymes leads to increased clearance of certain drugs, leading to decreased drug exposure and response ${ }^{30}$. CYP450 inducers could decrease the risk of hepatotoxicity of certain drugs. Examples of inducers are rifampin and phenobarbital. Induction of CYP3A by rifampin increases their metabolism and decreases their exposure,

requiring an increase in their dose ${ }^{32}$. Inhibition of CYP450 enzymes by endogenous or exogenous compounds leads to a decreased ability of the enzyme to clear the drug. CYP450 inhibitors can drastically increase the blood levels of various substrates of CYP450 enzymes, leading to toxicity. Inhibitors of CYP450 enzymes include azole antifungals; HIV protease inhibitors, such as ritonavir; and certain hepatitis $\mathrm{C}$ virus (HCV) drugs ${ }^{33}$.

\section{Phase II pathway}

During phase II drug metabolism, the drugs or metabolites from phase I pathways are enzymatically conjugated with a hydrophilic endogenous compound with the help of transferase enzymes. The most common phase II drugmetabolizing enzymes are UDP glucuronosyltransferases (UGTs), sulfotransferases (SULTs), N-acetyltransferases (NATs), glutathione S-transferases (GSTs), thiopurine Smethyltransferases (TPMTs), and catechol Omethyltransferases (COMTs) ${ }^{34}$.

\section{Uridine 5'-diphospho-glucuronosyltransferases}

Glucuronidation is a major phase II drug metabolism pathway, with approximately $40 \%$ to $70 \%$ of human endogenous and exogenous compounds conjugated to glucuronidated end products. Conjugated products are more hydrophilic and are readily excreted from the body.
In the cytoplasm, glucose-1 phosphate reacts with uridine triphosphate to form uridine diphosphate glucuronic acid (UDPGA), a cosubstrate and this is transferred into the ER by transmembrane proteins ${ }^{35}$. In the ER, UGT attaches UDPGA to the appropriate substrate by the nucleophilic attack, forming glucuronidated compounds. UGTs are members of a superfamily of protein, having a molecular weight in the range of $50 \mathrm{kDa}$ to $60 \mathrm{kDa}$, and structurally they have a catalytic domain and a C-terminal anchoring domain. Until now, 4 families of UGTs in humans have been identified: UGT1, UGT2, UGT3, and UGT8. UGT2s have been subdivided into UGT2A and UGT2B ${ }^{33,34}$

UGTs metabolize a wide range of compounds and their substrates also overlap with each other. Based on current knowledge, UGT1A1 is the highly expressed phase II enzyme in humans, which preferentially metabolizes bilirubin; UGT1A1 also metabolizes certain phenols and estradiols. Whereas UGT2B7 metabolizes opiates, UGT1A3, UGT1A9, and UGT2A1 metabolize carboxylic acids ${ }^{36}$. Various organs express UGTs; however, UGTs are normally highly expressed in the liver and gut. The functional activity of the UGTs is controlled by the number of enzymes available and the amount of co-substrate available to conjugate the drug or the metabolite. There are some drugs, such as phenobarbital and rifampin, which are known to increase the expression of UGTs and decreased drug exposure.

\section{Sulfotransferases}

SULTs are another important superfamily of phase II drugmetabolizing enzymes. Although they are not as highly expressed in the body as UGTs, they are essential for the metabolism of several endogenous compounds. They catalyze the reaction between $3^{\prime}$-phosphoadenosine 5'phosphosulphate (PAPS) and $\mathrm{N}, \mathrm{O}$, or $\mathrm{S}$ atoms in targeted compounds ${ }^{34}$. A wide range of endogenous compounds (steroids, catecholamine, serotonin, eicosanoids, retinol, and so forth), as well as exogenous compounds, are metabolized by SULTs. An endogenous compound like dopamine is almost entirely metabolized by SULTs. Expression of SULTs in humans occurs almost in every organ, most commonly found in the liver, gut, breast, lung, adrenal glands, kidney, blood cells, brain, and placenta. SULTs have 2 forms; one is metabolically very important and presents in the cytosol, the other one is membranebound and metabolically less important ${ }^{36}$. The function of the membrane-bound isoforms is to synthesize housekeeping substances rather than to metabolize endogenous or exogenous compounds.

Until now, 13 SULTs have been identified in humans and they have been divided into 4 families: SULT1, SULT2, SULT4, and SULT6. There are 9 members in the SULT1 family, which could be further subdivided into 4 subfamilies (1A1, 1A2, 1A3, 1A4; 1B1; $1 \mathrm{C} 1$ and 1C2; and 1E1). On the other hand, the SULT2 family has been divided into 2 subfamilies, SULT2A, and SULT2B ${ }^{7,8}$. Additionally, the SULT4 and SULT6 family contain only 1 member in each group, SULT4A1, and SULT6B1, respectively. So far, 
SULT1A1 is the most extensively studied sulfation enzyme, which metabolizes phenols, alcohols, and amines. SULT1A2 and SULT1A3 also metabolize amines; aromatic amines are the primary substrates for both of these isoforms. SULT1B1 is restricted to the metabolism of thyroids hormones and some small phenolic compounds ${ }^{37}$.

SULT1C1 metabolizes iodothyronines, and SULT1C2 metabolizes 4-nitrophenols. Furthermore, SULT1E1 has a special preference for the metabolism of estrogens, even though it has an affinity for other compounds as well. Various compounds are reported to be inhibitors of SULTs. Curcumin is a potent inhibitor of SULT1A1. Several medications can induce the expression of SULTs in human cells. Retinoic acid induces SULT1A1, SULT2A1, and SULT1E1 in hepatic carcinoma cells as well as in Caco-2 cells ${ }^{35}$. Methotrexate has been shown to have induction capability for various SULTs enzymes in human cells.

\section{$\mathrm{N}$-acetyltransferases}

Unlike other enzymes, products of NATs are sometimes more lipophilic instead of being more hydrophilic (metabolites of sulfonamides). In certain situations, the metabolite itself can become more toxic than the parent compound. Several enzymes increase the hydrophilicity of the metabolites as well as perform housekeeping activity, such as histone acetyltransferase that regulates the expression of genes in humans and other animals ${ }^{37}$. Acetyltransferases in humans are classified into 2 subfamilies: NAT1 and NAT2. All NATs are cytosolic enzymes and they use acetyl coenzyme $A$ as a cofactor for a metabolic reaction. Until now, 25 members of NAT1 and 27 members of NAT2 alleles have been identified in humans. NAT1 is a ubiquitous enzyme, expressed in almost all tissues. NATs have different substrate specificity and they do not overlap like other metabolizing enzymes. Paraaminobenzoic acid, para-aminosalicylic acid, and para amino glutamate are the main substrates for NAT1 in humans. On the other hand, sulfamethazine, isoniazid, hydralazine, and sulfonamides are the common substrates for NAT2. Polyphenolic compounds are believed as the main inhibitors of NATs. Compounds like caffeic acid, esculetin, quercetin, and genitin inhibit NAT1, whereas scopoletin and coumarin are the known inhibitors of NAT $2^{37,38}$.

\section{Glutathione S-transferases}

GSTs are ubiquitously present isozymes, found in almost all animal species. GSTs are also, important phase II drugmetabolizing enzymes, involved in the metabolism of exogenous and endogenous compounds. Additionally, GSTs are crucial for the detoxification of endogenously produced free radicals, hence protecting the body from oxidative stress. There are 2 superfamilies of GSTs, and members of both the groups have transferases activity ${ }^{37}$. One group is called soluble GSTs; they are found mostly in the cytosol, and recent studies have shown that mitochondria also contain soluble forms of GSTs. The second group of GSTs is called microsomal transferases, also called membrane-associated proteins in eicosanoid and glutathione metabolism(MAPEG) ${ }^{35}$.Soluble GSTs are again divided into 8 families based on their degree of sequence identity. These enzymes are distributed throughout the human body; the most heavily expressed organs are the liver, kidney, brain, heart, lung, and gut. The most commonly used inhibitors of GST include phenols, quinone, certain vitamin C derivatives, dopamine, and trans-retinoic acid. Induction of GSTs may also be beneficial in certain situations, which would help remove oxidative molecules that are generated in the body ${ }^{35,36}$.

\section{Thiopurine S-methyltransferases}

TPMT is an important enzyme, particularly in cancer chemotherapy; this enzyme catalyzes S-methylation of aromatic heterocyclic sulfhydryl compounds, including anticancer and immunosuppressive medications. Thiopurines 6-mercaptopurine, 6-thioguanine, and azathioprine are prodrugs. These drugs have to be metabolically converted to the active form by hypoxanthine phosphoribosyl transferases. The metabolites of hypoxanthine phosphoribosyl transferases are cytotoxic and exert anticancer activity; There has to be a delicate balance between cytotoxicity for anticancer action in cancer cells and normal cells. TPMT metabolizes those compounds to the non-toxic forms by methylation 37. TMPT is a cytosolic enzyme mostly found in the liver, kidney, and lung; additionally, human red blood cells also have a significant level of TMPT expression. Thiopurines are good substrates for the TPMT, which makes them important for cancer chemotherapy. Inhibition of TPMT activity may lead to the accumulation of toxic metabolites in the human body, causing other conditions, such as myelosuppression after azathioprine treatment. Naproxen, mefenamic, and tolfenamic acid are known to inhibit TPMT in a noncompetitive manner ${ }^{36,37}$.

\section{Catechol O-methyltransferases}

COMTs are the enzymes responsible for the transfer of a methyl group from S-adenosylmethionine to its substrate. This methylation is one of the major pathways for the metabolism of catecholamines and catechol estrogens, including neurotransmitters, such as dopamine, epinephrine, and norepinephrine as well as drugs that have catechol functional groups attached to their structure. COMT is mostly expressed in the postsynaptic neurons in the mammalian cells ${ }^{36,37}$. There are 2 forms of COMT: the soluble form, called S-COMT, and the membrane-bound form, called MB-COMT. Structurally, both S-COMT and MB-COMT share almost similar sequences, however, their substrate affinity and specificity vary significantly. Inhibition of COMT leads to the accumulation of its substrate, which is used as treatment strategies for Parkinson's diseases ${ }^{38}$.

\section{Phase III pathway}

Drug transporters are generally transmembrane proteins that facilitate the transport of large and/or ionized molecules in and out of the cells. Phase III pathway is 
classified into 2 main superfamilies: ATP-binding cassette $(A B C)$ and solute carrier (SLC) transporters. $A B C$ transporters are dependent on the energy (ATP) consumption to actively uptake or efflux the drug from one side of the cell membrane to another, whereas SLCs facilitates the passage of certain solutes (eg, sugars and amino acids) across the membrane and actively transport other solutes against their electrochemical gradients by coupling the process with other solute or ion ${ }^{37,38}$. They are present in many locations, such as the liver, kidney, intestine, and brain. Conceptually, uptake transporters help in transferring the molecules into the cells and efflux transporters pump them outside the cell. In the liver, the main uptake transporters are Na1-taurocholate cotransporting polypeptide (NTCP), organic cation transporter 1 (OCT1), organic anion transporter 2 (OAT2), and organic anion-transporting polypeptides ${ }^{39}$. The hepatic efflux transporters are multidrug resistance protein 1 (MDR1) (also known as P-glycoprotein [P-gp] and $A B C$ subfamily $B$ member 1 [ABCB1]), bile salt export pump (BSEP) (also known as ABC subfamily B member 11 [ABCB11]), and multidrug resistance-associated protein (MRP) $2^{38,39}$.

\section{Sites of drug metabolism}

\section{Liver}

The liver is the major organ for metabolic processes which includes two phases that are phase I and phase II drug metabolic processes. The entry of drug molecules into the hepatocytes and out into the bile is performed by drug transporters. The drug transporters also have a role in transporting the large and charged compounds. Some drugs secreted in the bile are reabsorbed back from the intestine. And some metabolites can be converted back to the drug by b-Glucuronidase in the gut and can be reabsorbed. This process is known as enterohepatic circulation, a process that persists the drug in the body ${ }^{37,40}$. D and Da are two different drugs that have different clearance pathways. In the liver, the drug $D$ is metabolized by the phase I pathway followed by the conjugation process by phase II enzymes, and finally effluxed into bile by the transporter. In this scenario, drug Da is transported into the hepatocyte through OAT then pumped out by MDR1 without any chemical modification to the drug molecule ${ }^{40}$. First pass metabolism can occur in both liver and gut when the drugs are administered through the oral route. The extension of oral bioavailability of various drugs leads to liver diseases. In patients with liver cirrhosis, there is an increase in the severity of the drug-induced liver injury. In the HCV patient population, it is advised to closely monitor drug dosing after liver transplantation, especially when HCV therapies are used to prevent any recurrence.

\section{Gut}

The primary function of the intestinal tract is the absorption of nutrients, electrolytes, and drug molecules. The gut also has an important role in metabolic functions, however, which can have an impact on the systemic bioavailability of certain therapeutic agents. The orally administered drugs are not completely absorbed from the gut lumen and move into the enterocytes, then move into the portal circulation, then to the liver, and finally into the systemic circulation ${ }^{38}$. The systemic availability (F) of a drug is defined as, the administered dose of drugs that reaches the systemic circulation that is the product of fraction absorbed (Fab), fractions escaping gut metabolism $(\mathrm{Fg})$, and the fraction escaping hepatic metabolism (Fh) before reaching the systemic circulation, this can be expressed as, $\mathrm{F}=\mathrm{Fab} \times \mathrm{Fg} \times \mathrm{Fh}^{40}$. The human gut expresses a large portion of a phase I and phase II drug-metabolizing enzymes, including CYP450s and UGTs. The human small intestine expresses appreciable amounts of various CYP450 enzymes, contributing to the oxidation of several xenobiotics ${ }^{39}$. Among all the CYP450s, CYP3A4 is the most abundant CYP450 enzyme, contributes to bile acid detoxification, the termination of action of steroid hormones, and elimination of phytochemicals in food and the majority of medicine ${ }^{41}$. The small intestine expresses more than 50 CYP450 enzymes, but the CYP1A2, CYP2C9, CYP2C19, CYP2D6, CYP3A4, and CYP3A5 enzymes metabolize 90 percent of drugs. The amount of expression ofCYP450 in the intestine varies from $20 \mathrm{pmol} / \mathrm{g}$ to 210 $\mathrm{pmol} / \mathrm{g}$ of the tissues, indicating a large interpersonal variability. Additionally, the expression of the metabolic enzymes also is not uniform throughout the intestine. The duodenum, jejunum, and ileum are the places where expression is maximum, the expression level goes down further down in the intestine. UGTs are a superfamily of phase II conjugating enzyme expressed in the small intestine as well as in the liver and kidney. The subfamilies of UGT1A, UGT2B predominantly expressed in the intestine as well as in the liver and kidney. The hepatic UGTs, include UGT1A1, UGT1A6, UGT1A7, UGT1A8, UGT1A9, and UGT1A10. Among the human UGTs, UGT1A1 is of particular clinical significance due to its unique activity in the conjugative detoxification of bilirubin ${ }^{40,41}$. The significance of intestinal UGTs is exemplified by raloxifene, a selective estrogen receptor modulator used for the treatment of osteoporosis and breast cancer. The poor bioavailability of raloxifene occurs when the orally administered raloxifene is metabolized in the intestine by UGT1A8, UGT1A10, andUUGT1A1.The localization of uptake and efflux transporters in organs responsible for drug biotransformation and excretion 40,41 . The drugmetabolizing enzymes and transporters could mediate and alter the overall systemic availability of some drugs. Among all the efflux transporters, human gut expresses-gp (MDR1), MRPs, and BCRP (breast cancer resistance protein), which are known to be responsible for most of the efflux function ${ }^{41}$. At the luminal site, the expression of the efflux transporters increases with lower bioavailability of the drug, whereas overexpression at the abluminal site may increase systemic availability. Transporters are the entry and exit point of drugs which prevents the toxins or unwanted substances from entering into blood. The uptaking of drugs into the cell is facilitated by influx transporters are expressed at the luminal site as well as in 
the abluminal site. OATP3A1, OATP4A1, OCT1, and OCT2are were expressed at the abluminal site as well ${ }^{39,41}$. By contrast, the efflux transporters may be responsible to export the drug to outside the cell.

\section{Kidney}

The important function of the kidney is the elimination of toxins and play a role in an excretory organ but have a lesser contribution in terms of overall drug metabolism. The main functional unit of the kidney is nephrons ${ }^{40,41}$. At first, the drugs are normally filtered by the glomerulus and excreted, and secreted at the proximal tubules, and reabsorbed by the tubules. Phase III transporters play a critical role in actively secreting drug molecules against their electrochemical gradients. Kidneys do contribute to the metabolism of some endogenous compounds and xenobiotics ${ }^{41}$. For example, kidneys activate 25 hydroxyvitamin $D$ to the hormone 1,25-dihydroxy vitamin D3 by CYP27B1, then deactivate it by CYP24A1 ${ }^{40,42}$. Cyclosporine is converted to its metabolites in the kidney by CYP3A enzymes in the kidney and this may contribute to cyclosporine mediated nephrotoxicity.

\section{Potential antiviral drugs for sars-cov-2}

Currently, there are no antiviral drugs or vaccines for SARSCoV-2. The development of a safe vaccine may take time. Different potential preclinical antiviral drugs are alphainterferon, chloroquine phosphate, arabinol, remdesivir, lopinavir/ritonavir, and anti-inflammatory drugs ${ }^{10}$.

\section{Lopinavir/ritonavir}

Lopinavir and ritonavir are protease inhibitors. They both play an important role in the treatment of chronic HIV. When Lopinavir was combined with Ritonavir, it was found to be effective against patients infected with SARS-CoV1and MERS-CoV. They interacted well with the residues at the active sites of SARS-CoV-2 3CLpro. Lopinavir had lower water accessibility when compared to Ritonavir at 3CLpro active site. Ritonavir was found to have higher bonding efficiency and atomic contacts. They also had a higher number of key binding residues. The adverse effect of Lopinavir/ritonavir includes gastrointestinal distress such as nausea and diarrhea, and hepato-toxicity ${ }^{43}$. One trial on Lopinavir/ritonavir in adults with severe Covid-19 stated that no beneficial effects were using these drugs ${ }^{10}$. More clinical studies are needed to use them safely to treat patients with Covid-19.

\section{Choloroquine and hydroxychloroquine}

Chloroquine and hydroxychloroquine are said to play an important role to treat and prevent SARS-CoV-2. Hydroxychloroquine is more soluble than chloroquine because it has an $\mathrm{N}$-hydroxy Methyl side chain in place of the $\mathrm{N}$-diethyl group of chloroquine and is also less toxic when compared to chloroquine. The SARS-CoV-2 enters the cell by binding to the ACE-2 receptor. Chloroquine inhibits terminal glycosylation and prevents the virus from binding to the ACE-2 receptor. Chloroquine combined with hydroxychloroquine can increase the $\mathrm{pH}$ of intracellular components which results in dysfunction leading to defective proteins. It is not clear how an increase in $\mathrm{pH}$ affects the integrity of SARS-CoV-2. However, Chloroquine and hydroxychloroquine have several mechanisms of actions against SARS-CoV-2 ${ }^{42}$. A review by Juurlink et al. ${ }^{43}$ stated that the use of either chloroquine or hydroxychloroquine and azithromycin for treatment or prevention of SARS-CoV-2 infection needed careful patient selection and monitoring. In another study by Zhou et al. ${ }^{44}$, the use of oral chloroquine phosphate tablets on COVID 19 patients induced adverse effects on patients. More clinical studies are required for the use of Chloroquine or hydroxychloroquine on COVID 19 patients.

\section{Remdesivir}

Remdesivir also is known as GS-5734 is a monophosphate prodrug that was used to treat Ebola virus disease and Marburg virus infections. They were also used to treat MERS and SARS viruses. Remdesivir when metabolized into an active form can inhibit the production of viral RNA. Currently, this drug has been tested in the laboratory on cultured cells and mouse and non-human primate (NHP) models, demonstrated its efficacy as a valuable antiviral drug against SARS/MERS-CoV viruses ${ }^{10}$. One preliminary study conducted by John $\mathrm{H}$. Beigel et al. ${ }^{45}$ on Remdesivir for the Treatment of Covid-19 stated that patients who are hospitalized with Covid-19 require supplemental oxygen therapy. Using Remdesivir alone didn't show any beneficial results and also the mortality rate was high. However, the author stated that Combining Remdesivir with other antiviral drugs or therapeutic approaches can increase efficacy.

\section{Ribavirin}

Ribavirin is an antiviral drug. They are used for the treatment of Hepatitis C virus, viral hemorrhagic fevers, and Respiratory syncytial virus. They inhibit the nucleoside and also arrest the synthesis and capping of viral mRNA ${ }^{10}$. They also block the RNA metabolism necessary for viral replication. In one study by Elfiky ${ }^{46}$, had targeted antiRNA-dependent RNA polymerase (RdRp) drugs, sofosbuvir, ribavirin, sofosbuvir, remdesivir, and IDX-184. This study concluded that these drugs could inhibit the protein function of SARS-CoV-2 and also eliminate the virus. Their safety profile has to be explored more.

\section{Ivermectin}

Ivermectin is a member of the Avermectin family. This compound is produced from the soil microorganisms streptomyces avermitilis. This compound has antiviral, antibacterial, and anti-cancer activity. Its mechanisms of action involve the opening of glutamate-gated Tebrates and GABA-gated chloride channel leading to increased conductance of chloride ions and causing subsequent motor paralysis in the parasite. They are found to be active against several viruses like the Zika virus, Influenza- A virus, Venezuelan equine, encephalitis virus. Etc. In SARS-CoV-2 Ivermectin prevents the binding of imp $\alpha / \beta 1$ to viral protein and prevents the viral protein from entering the 
nucleus. According to the study of Caly et al, vero-hsLAM cells were treated with Ivermectin after 2 hrs of SARS COV2 infection which resulted in $\sim 5000$ fold reduction in viral RNA after $48 \mathrm{hrs}$. Based on these studies, Ivermectin is considered as a useful drug for COVID $19^{42}$.

\section{Arbidol}

Arbidol is an antiviral drug used for the treatment of upper respiratory tract infections caused by the influenza virus. They prevent the virus from binding to the human cells. Some studies have confirmed that Arbidol is effective against SARS-CoV-2. A group of scientists has found out that $10-30 \mu$ l of Arbidol can inhibit SARS-CoV-2 effectively up to 60 times and also can inhibit the pathological effects of the virus. It has been reported that when arbidol was given to adults with COVID 19 at a dose of 200mg three times a day which didn't exceed 10 days showed effective improvement in symptoms ${ }^{9}$.

\section{Tocilizumab}

Tocilizumab is a recombinant humanized monoclonal antibody. It belongs to the Immunoglobulin G1 (IgG1 class), directed against both the soluble interleukin6 (IL-6) receptor (sIL-6R) and the receptor bound to the membrane (mIL-6R). IL-6 is a cytokine with proinflammatory features and is involved in many biological processes (as T-cells activation, fibrosis of tissues, metabolism of lipids). A group of researchers treated 21 patients affected by COVID-19 pneumonia with tocilizumab (400 mg/iv) and obtained positive results and suggested a randomized clinical trial in the future ${ }^{10}$. In one study by $\mathrm{Xu}$ et al. ${ }^{47}$ tocilizumab improved clinical symptoms effectively. It repressed the deterioration of severe COVID 19 patients. Tocilizumab seems to be an effective treatment for COVID $^{19}$.

\section{Ongoing clinical researches on SARS CoV-2}

Currently, there is a multicenter randomized clinical trial going on which has been proposed by the University of Seoul, Korea. This clinical trial is done on patients with mild COVID-19 to compare the efficacy of lopinavir/ritonavir given every $12 \mathrm{~h}$ for7-10 days at an amount of $400 \mathrm{mg} / 100 \mathrm{mg}$ twice daily with hydroxychloroquine (400mg twice daily). Another randomized clinical trial is testing the combination of lopinavir/ritonavir (400mg/100mg twice daily), ribavirin $(400 \mathrm{mg} / 100 \mathrm{mg}$ twice daily), and interferon beta-1b ( 8 million $I U$ in 0 to 3 subcutaneous injections) compared with to lopinavir/ritonavir alone (NCT04276688). There is also a Phase III clinical trials going on currently, on patients with COVID 19 using tocilizumab. Under the coordination of the legal promoter- IRCCS Institute Nazionale Tumori, IRCCS, Fondazione G. Pascale of Naples, a multicentre study will be conducted on 330 patients with COVID-19 pneumonia to test the efficacy and tolerability of tocilizumab in patients with severe COVID-19 pneumonia ${ }^{10}$. In one study Natural products spotlighted anti-COVID-19 phytochemicals. The synergy of virtual screening docking and modeling is indispensable for exploiting the chemical diversity of natural products. The molecular modifications of natural products through Michael acceptor groups may be a new strategy for $3 C L$ proinhibitors ${ }^{48}$.

A synthetic cannabinoid drug has been designed to treat a life-threatening lung condition called Acute Respiratory Distress Syndrome (ARDS) found in several novel coronavirus cases. The FDA has approved the phase I clinical trials for this drug. ARDS is triggered by over secretion of cytokines which causes more inflammation in the body. ARDS-003 is the drug that is going to the clinical trials. This drug will lessen the cytokine production and thus prevent the development of ARDS. This drug works by binding to CB2 receptors, one of two main receptors in the endocannabinoid system which modulate inflammation and cytokine activity. CB2 receptors don't bring on a psychoactive high. Phase I clinical trials will begin in December to determine the safety of the drug in humans ${ }^{49}$.

\section{CONCLUSION}

Currently, we distinguish a huge and diverse number of physiological receptors which can be defined into receptor super-families based on their pharmacology and molecular properties. These receptors are now the focus of many of the multinational, multibillion-dollar pharmaceutical industry. The receptors are not fixed compounds but then they are subjected to regulation of receptor function. Heterodimer and oligomers open new outlooks or viewpoints for GPCRs from both the functional and the pharmacological point of view. The susceptibility of drugs binding to different classes of receptors provides an opportunity to develop new drug molecules.

Drug metabolism may result in detoxification or bioactivation of drugs. The detoxification process involves two phases of drug metabolism: phase- I convert highly lipophilic drug molecules to polar metabolites, whereas in phase-II involves conjugation reactions. Metabolisms include a variety of reactions such as oxidation, reduction, hydroxylation, dealkylation, etc. In detoxification reactions, CYP enzymes can result in the formation of reactive metabolites, which lead to toxicity and drug-drug interactions. Most of the metabolic reactions are carried out by CYP enzymes. Lack of these enzymes will sometimes make the drug low efficient.

Though there are no vaccines or a particular drug to treat COVID 19. The above described potential Antiviral drugs are found to be effective in preclinical trials. However more clinical researches are needed to study the safety profile and efficacy of the drugs to treat patients with SARS-CoV-2.

\section{Funding: Nil}

Authors Contributions: All the authors have contributed equally.

Conflict of Interests: Declared none 


\section{REFERENCES}

1. Langley JN. On the reaction of cells and of nerve-endings to certain poisons, chiefly as regards the reaction of striated muscle to nicotine and to curari. The Journal of physiology. $1905 \mathrm{Dec}$ 30;33(4-5):374, PMID: 16992819.

Doi: 10.1113/jphysiol.1905.sp001128;

2. Copeland RA, Pompliano DL, Meek TD. Drug-target residence time and its implications for lead optimization. Nature reviews Drug discovery. 2006 Sep;5(9):730-9, Doi:10.1038/nrd2082; PMID:16888652.

3. Swinney DC. The role of binding kinetics in therapeutically useful drug action. Current opinion in drug discovery \& development. 2009 Jan;12(1):31; PMID: 19152211

4. Lu H, Tonge PJ. Drug-target residence time: critical information for lead optimization. Current opinion in chemical biology. 2010 Aug 1;14(4):467-74, PMID: 20663707. doi:10.1016/j.cbpa.2010.06.176;

5. Benedetti MS, Whomsley R, Poggesi I, Cawello W, Mathy FX, Delporte ML, Papeleu P, Watelet JB. Drug metabolism and pharmacokinetics. Drug metabolism reviews. 2009 Aug 1;41(3):344-90, Doi: $10.1080 / 10837450902891485 ;$ PMID: 19601720.

6. Ionescu C, Caira MR. Drug metabolism: current concepts. Dordrecht (Netherlands): Springer; 2005; Available: https://bok.cc/book/2205921/660c1a.

7. Al-Sadeq DW, Nasrallah GK. The incidence of the novel coronavirus SARS-CoV-2 among asymptomatic patients: a systematic review. International Journal of Infectious Diseases. 2020 Sep 1;98:372-80, Doi: 10.1016/j.ijid.2020.06.098; PMID: 32623083.

8. Singhal T. A review of coronavirus disease-2019 (COVID-19). The Indian Journal of Pediatrics. 2020 Mar 13:1-6, doi :10.1007/s12098-020-03263-6; PMID: 32166607.

9. Li H, Yang L, Liu FF, Ma XN, He PL, Tang W, Tong XK, Zuo JP. Overview of therapeutic drug research for COVID-19 in China. Acta Pharmacologica Sinica. 2020 Sep;41(9):1133-40, doi:10.1038/s41401-020-0438-y; PMID: 32555446.

10. BIMONTE S, CRISPO A, AMORE A, CELENTANO E, CUOMO A, CASCELLA M. Potential Antiviral Drugs for SARS-Cov-2 Treatment: Preclinical Findings and Ongoing Clinical Research. In Vivo. 2020 Jun 1;34(3 suppl):1597-602, doi: 10.21873/invivo.11949; PMID: 32503817 .

11. Drug-Receptor Interactions By Abimbola Farinde Ph.D., PharmD, Columbia Southern University, Orange Beach, ALLast full review/revision Jun 2019| Content last modified Jun 2019; Available : https://www.merckmanuals.com/professional/clinicalpharmacology/pharmacodynamics/dose-response-relationships.

12. Limbird LE. The receptor concept: a continuing evolution. molecular interventions. 2004 Dec 1;4(6):326, doi: 10.1124/mi.4.6.6; PMID: 15616162.

13. Lefkowitz RJ. Seven transmembrane receptors-A brief personal retrospective. Biochimica et Biophysica Acta (BBA)Biomembranes. 2007 Apr 1;1768(4):748-55, Doi: 10.1016/j.bbamem.2006.11.001; PMID: 17173855.

14. Humphrey PP, Barnard EA. International Union of Pharmacology. XIX. The IUPHAR receptor code: a proposal for an alphanumeric classification system. Pharmacological reviews. 1998 Jun 1;50(2):271-8, PMID: 9647868

15. Wise A, Gearing K, Rees S. Target validation of G-protein coupled receptors. Drug discovery today. 2002 Feb 15;7(4):235-46, Doi:10.1016/S1359-6446(01)02131-6; PMID: 11839521.

16. Foord SM, Jupe S, Holbrook J. Bioinformatics and type II G-proteincoupled receptors, September 2002. Biochemical Society
Transactions 30 (4): 473-9, Doi:10.1042/bst0300473, PMID: 12196118.

17. Attwood TK, Findlay JB. Fingerprinting G-protein-coupled receptors. Protein Engineering, Design and Selection. 1994 Feb 1;7(2):195-203, Doi:10.1093/protein/7.2.195.

18. Farran B. An update on the physiological and therapeutic relevance of GPCR oligomers. Pharmacological research. $2017 \mathrm{Mar}$ 1;117:303-27, Doi: 10.1016/j.phrs.2017.01.008.

19. Guidolin D, Marcoli M, Tortorella C, Maura G, Agnati LF. G proteincoupled receptor-receptor interactions give integrative dynamics to intercellular communication. Reviews in the neurosciences. 2018 Sep 25;29(7):703-26, Doi:10.1515/revneuro-2017-0087.

20. Kucharski R, Mitri C, Grau Y, Maleszka R. Characterization of a metabotropic glutamate receptor in the honeybee (Apis mellifera): implications for memory formation. Invertebrate Neuroscience. 2007 Jun 1;7(2):99-108, Doi: 10.1038/35039564; PMID: 11069170.

21. Lee SM, Booe JM, Pioszak AA. Structural insights into ligand recognition and selectivity for classes $A, B$, and C GPCRs. European journal of pharmacology. 2015 Sep 15;763:196-205, Doi: 10.1016/j.ejphar.2015.05.013; PMID: 25981303.

22. Bayburt TH, Leitz AJ, Xie G, Oprian DD, Sligar SG. Transducin activation by nanoscale lipid bilayers containing one and two rhodopsins. Journal of biological chemistry. 2007 May 18;282(20):14875-81, Doi: 10.1074/jbc.M701433200; PMID: 17395586.

23. Whorton MR, Bokoch MP, Rasmussen SG, Huang B, Zare RN, Kobilka B, Sunahara RK. A monomeric G protein-coupled receptor isolated in a high-density lipoprotein particle efficiently activates its $\mathrm{G}$ protein. Proceedings of the National Academy of Sciences. 2007 May 1;104(18):7682-7, Doi: 10.1073/pnas.0611448104; PMID: 17452637.

24. Kuszak AJ, Pitchiaya S, Anand JP, Mosberg HI, Walter NG, Sunahara RK. Purification and Functional Reconstitution of Monomeric $\mu$ Opioid Receptors ALLOSTERIC MODULATION OF AGONIST BINDING BY Gi2. Journal of Biological Chemistry. 2009 Sep 25;284(39):26732-41; Doi: 10.1074/jbc.M109.026922; PMID: 19542234.

25. Goupil E, Laporte SA, Hébert TE. Functional selectivity in GPCR signaling: understanding the full spectrum of receptor conformations. Mini-Rev Med Chem,2012, 12:817-30, Doi: 10.2174/138955712800959143; PMID: 22681252.

26. Pharmacology Corner: Drug-Receptor Interactions, Aegis Sciences Corporation,2015. Available: www.aegislabs.com/drugreceptor

27. Zidar DA, Violin JD, Whalen EJ, Lefkowitz RJ. Selective engagement of $G$ protein-coupled receptor kinases (GRKs) encodes distinct functions of biased ligands. Proc Natl Acad Sci USA,2009 106:9649-54. 10.1073/pnas.0904361106, Doi: 10.1073/pnas.0904361106; PMID: 19497875.

28. Drug-Receptor Interactions By Abimbola Farinde PhD, PharmD, Columbia Southern University, Orange Beach, Jun 2019; Available: https://www.merckmanuals.com/professional/clinicalpharmacology/pharmacodynamics/drug\%E2\%80\%93receptorinteractions.

29. Charles Ophardt, Professor Emeritus, Elmhurst College; Drug Receptor Interactions Last updated, Aug 11, 2020; Available: https://chem.libretexts.org/Bookshelves/Biological_Chemistry/Su pplemental_Modules_(Biological_Chemistry)/Pharmaceuticals/Dr ug_Receptor_Interactions.

30. Olsen L, Oostenbrink C, Jorgensen FS. Prediction of cytochrome P450 mediated metabolism. Adv Drug Deliv Rev, 2015;86:61-71, Doi: 10.1016/j.addr.2015.04.020; PMID: 25958010.

31. Mittal B, Tulsyan S, Kumar S, Mittal RD, Agarwal G. Cytochrome P450 in cancer susceptibility and treatment. InAdvances in clinical 
chemistry 2015 Jan 1 (Vol. 71, pp. 77-139). Elsevier publications, Doi: 10.1016/bs.acc.2015.06.003; PMID: 26411412.

32. Terada T, Hira D. Intestinal and hepatic drug transporters: pharmacokinetic, pathophysiological and pharmacogenetic roles. J Gastroenterol, 2015;50(5):508-19, Doi: 10.1007/s00535-0151061-4; PMID: 25773773.

33. Parikh ND, Levitsky J. Hepatotoxicity and drug interactions in liver transplant candidates and recipients. Clin Liver Dis, 2013;17(4):737-47, $\quad x-x i, \quad$ Doi: 10.1016/j.cld.2013.07.013; PMID: 24099028.

34. Tischer S, Fontana RJ. Drug-drug interactions with oral antiHCVagents and idiosyncratic hepatotoxicity in the liver transplant setting. J Hepatol, 2014;60(4):872-84, Doi: 10.1016/j.jhep.2013.11.013; PMID: 24280292.

35. Feghali M, Venkataramanan R, Caritis S. Pharmacokinetics of drugs in pregnancy. Semin Perinatol, 2015;39(7):512-9, Doi: 10.1053/j.semperi.2015.08.003; PMID: 26452316

36. Woolsey SJ, Mansell SE, Kim RB, Tirona RG, Beaton MD. CYP3A activity and expression in nonalcoholic fatty liver disease. Drug Metabolism and Disposition. 2015 Oct 1;43(10):1484-90, Doi: 10.1124/dmd.115.065979; PMID: 26231377

37. Schuck RN, Zha W, Edin ML, Gruzdev A, Vendrov KC, Miller TM, Xu Z, Lih FB, DeGraff LM, Tomer KB, Jones HM. The cytochrome P450 epoxygenase pathway regulates the hepatic inflammatory response in fatty liver disease. PloS one. 2014 Oct 13;9(10):e110162, Doi: 10.1371/journal.pone.0110162; PMID: 25310404.

38. Ladda MA, Goralski KB. The effects of CKD on cytochrome P450mediated drug metabolism. Adv Chronic Kidney Dis, 2016;23(2):67-75, Doi: 10.1053/j.ackd.2015.10.002; PMID: 26979145.

39. Joy MS, Frye RF, Nolin TD, Roberts BV, La MK, Wang J, Brouwer KL, Dooley MA, Falk RJ. In vivo alterations in drug metabolism and transport pathways in patients with chronic kidney diseases. Pharmacotherapy: The Journal of Human Pharmacology and Drug Therapy. 2014 Feb;34(2):114-22, doi: 10.1002/phar.1347; PMID: 24038489.

40. Li M, Zhao Y, Humar A, Tevar AD, Hughes C, Venkataramanan R. Pharmacokinetics of drugs in adult living donor liver transplant patients: regulatory factors and observations based on studies in animals and humans. Expert Opinion on Drug Metabolism \& Toxicology. $2016 \quad$ Mar 3;12(3):231-43, Doi: $10.1517 / 17425255.2016 .1139575$.

41. Brüggemann RJ, Alffenaar JW, Blijlevens NM, Billaud EM, Kosterink JG, Verweij PE, Burger DM, Saravolatz LD. Clinical relevance of the pharmacokinetic interactions of azole antifungal drugs with other coadministered agents. Clinical Infectious Diseases. 2009 May 15;48(10):1441-58, Doi: 10.1086/598327.

42. Bharad VR, Deshmukh OR, Wagh SM, Folane P, Kale RH, Biyani KR. The Review on current treatments and therapies on COVID-19. Asian Journal of Pharmaceutical Research and Development. 2020 Aug 15;8(4):122-9, Doi: 10.22270/ajprd.v8i4.800.

43. Juurlink DN. Safety considerations with chloroquine, hydroxychloroquine and azithromycin in the management of SARS-CoV-2 infection. CMAJ. 2020 Apr 27;192(17):E450-3, Doi: 10.1503/cmaj.200528; PMID: 32269021.

44. Li L, Zhou B, Zhang J, Liu Y, Hong W, Jian F, Wang M, Tan J, Cai W, Zhang F. Safety review of 42 cases of COVID-19 treated with lowdose chloroquine, Research Square, Doi: 10.21203/rs.3.rs22918/v1.

45. Beigel JH, Tomashek KM, Dodd LE, Mehta AK, Zingman BS, Kalil AC, Hohmann E, Chu HY, Luetkemeyer A, Kline S, de Castilla DL. Remdesivir for the treatment of Covid-19-preliminary report. The New England journal of medicine. 2020 May 22, Doi: 10.1056/NEJMoa2007764; PMID: 32445440.

46. Elfiky A. A. Anti-HCV, nucleotide inhibitors, repurposing against COVID-19. Life Sciences, 248: 117477, 2020, Doi: 10.1016/j.Ifs.2020.117477; PMID: 32119961.

47. Xu X, Han M, Li T, Sun W, Wang D, Fu B, Zhou Y, Zheng X, Yang Y, Li $X$, Zhang $X$. Effective treatment of severe COVID-19 patients with tocilizumab. Proceedings of the National Academy of Sciences. 2020 May 19;117(20):10970-5, DOI: 10.1073/pnas.2005615117.

48. Pawar RS, Dimri M, Maithani A. Anti-COVID-19 natural products are spotlights for drug discovery and development. Asian Journal of Pharmaceutical Research and Development. 2020 Aug 15;8(4):88-90, Doi: 10.22270/ajprd.v8i4.785.

49. Ralph Ellis. FDA Approves Clinical Trials for Cannabinoid Drug Designed to Reduce COVID Lung Inflammation [online]. Available: https://www.medscape.com/viewarticle/936161

Source of Support: None declared.

Conflict of Interest: None declared.

For any question relates to this article, please reach us at: editor@globalresearchonline.net New manuscripts for publication can be submitted at: submit@globalresearchonline.net and submit_ijpsrr@rediffmail.com 